\title{
EXPLICIT FORMULAS FOR INFINITELY MANY SHIMURA CURVES IN GENUS 4
}

\author{
SAMUEL GRUSHEVSKY AND MARTIN MÖLLER
}

\begin{abstract}
In this paper we construct infinitely many Shimura curves contained in the locus of Jacobians of genus four curves. All Jacobians in these families are $\mathbb{Z} / 3$ covers of varying elliptic curves that appear in a geometric construction of Pirola, and include an example of a Shimura-Teichmüller curve that parameterizes Jacobians that are suitable $\mathbb{Z} / 6$ covers of $\mathbb{P}^{1}$. We compute explicitly the period matrices of the Shimura curves we construct using the original construction of Shimura for moduli spaces of abelian varieties with automorphisms.
\end{abstract}

\section{INTRODUCTION}

A well-known question in relating the geometric and arithmetic properties of symmetric domains is the following problem expectation of Oort (posed in [Oor97], see [MO13]): for $g$ sufficiently large, does the moduli space $\mathcal{A}_{g}$ of complex principally polarized abelian varieties contain any Shimura subvariety contained generically in the Torelli locus $\mathcal{J}_{g}$ - the locus of Jacobians of smooth genus $g$ curves? A finite number of examples of Shimura curves contained in the Torelli locus have been constructed by de Jong, Moonen, Mumford, Noot, Oort, and others, most of them arising as Galois covers of $\mathbb{P}^{1}$ with branch points varying - see the survey [MO13] for the history of the problem, details, and references; see also [FGP14] for more examples arising as non-abelian Galois covers.

From the other direction, a lot of work has been devoted to proving that for $g$ sufficiently large the Torelli locus contains no special subvarieties. The latest progress in this direction has been made by Chen, Lu and Zuo, who proved that there do not exist Shimura curves of Mumford type, or of maximal variation, or contained in the locus of hyperelliptic Jacobians, for some explicit low bounds on $g$, see [LZ13, CLZ15].

Research of the first author is supported in part by National Science Foundation under the grants DMS-1201369 and DMS-1501265. Research of the second author is supported in part by ERC-StG-257137. 
Our modest contribution in this note is an explicit construction - by writing down families of period matrices — of infinitely many Shimura curves contained in $\mathcal{J}_{4}$.

Theorem 1. For a dense set of points $z_{1}, z_{2}$ in a 2-dimensional complex ball, the one-parameter family of $4 \times 8$ period matrices, given by

$$
\left(Z_{1} \mid Z_{2}\right)=\left(\begin{array}{cccc}
3 \tau & 0 & 3 \tau+3 & 0 \\
0 & Z_{1}^{(3)}\left(z_{1}, z_{2}\right) & 0 & Z_{2}^{(3)}\left(z_{1}, z_{2}\right)
\end{array}\right) B^{-1}
$$

where $\tau \in \mathbb{H}$ is the parameter for the family, where $B$ is given by (2), and $Z_{1}^{(3)}, Z_{2}^{(3)}$ are given by Proposition 7 , defines a Shimura curve contained generically in the locus of Jacobians of smooth curves of genus 4.

Our examples include the Shimura-Teichmüller curve of genus 4 ([Möl11])) obtained for the values

$$
z_{1}=\frac{-2 \zeta^{3}+\zeta^{2}+\zeta-3}{2}, \quad z_{2}=3^{-1 / 4} \frac{\zeta^{3}-2 \zeta^{2}+1}{2} .
$$

These Shimura curves are not of maximal degeneration or of Mumford type in the sense of [LZ13].

Unlike our purely analytic construction of infinitely many Shimura curves contained in the locus of hyperelliptic Jacobians of genus three [GM13], the construction in this note is geometric, by using $\mathbb{Z} / 3$ Galois covers of elliptic covers, and the associated Prym map studied by Pirola $([$ Pir92]).

We announced our results at several talks starting in February 2014, including at Oberwolfach, Paris Jussieu, and Roma Tre. At the last stage of preparing our manuscript, the preprint of Frediani, Penegini, Porru [FPP15] appeared, which studies much more generally under what conditions families of covers of elliptic curves may lead to Shimura curves. They independently discovered our examples, and much more, while we are able to compute the period matrices explicitly using the Shimura description.

Notation. We denote by $\mathcal{M}_{g}$ the moduli space of smooth complex curves, and by $\mathcal{A}_{g}$ the moduli space of complex principally polarized abelian varieties (ppav). We denote by Jac $: \mathcal{M}_{g} \rightarrow \mathcal{A}_{g}$ the Torelli map that sends the curve to its Jacobian. The main question we study here is the existence of Shimura curves that are contained generically in $\mathcal{J}_{g}:=\operatorname{Jac}\left(\mathcal{M}_{g}\right)$. A Kuga fiber space is an inclusion $j$ of a $\mathbb{Q}$-algebraic group $G$ into $\operatorname{Sp}(2 g, \mathbb{Q})$, such that an arithmetic lattice $\Gamma \subset G_{\mathbb{R}}$ maps to $\operatorname{Sp}(2 g, \mathbb{Z})$ and such that the intersection of the maximal compact subgroup $U(g) \subset \operatorname{Sp}(2 g, \mathbb{R})$ with $j\left(G_{\mathbb{R}}\right)$ is a maximal compact subgroup 
$K_{\mathbb{R}}$ of $G_{\mathbb{R}}$. We identify a Kuga fiber space with the subvariety of the moduli stack

$$
\Gamma \backslash G_{\mathbb{R}} / K_{\mathbb{R}} \hookrightarrow \operatorname{Sp}(2 g, \mathbb{Z}) \backslash \operatorname{Sp}(2 g, \mathbb{R}) / U(g)=\mathcal{A}_{g} .
$$

A Shimura subvariety of $\mathcal{A}_{g}$ is the image of such an inclusion that moreover contains a CM point. One-dimensional Kuga fiber spaces $\Gamma \backslash G_{\mathbb{R}} / K_{\mathbb{R}}$ are called Kuga curves, one-dimensional Shimura varieties are called Shimura curves.

We refer to [MO13] for a detailed discussion of the history and importance of the problem, its relationship with the André-Oort and Coleman conjectures, the current status, and further references.

In Section 1 we review Pirola's construction and explain how it gives examples of Shimura curves. In Section 2 we compute the period matrix of the Shimura-Teichmüller curve in genus 4. explicitly. In Section 3 we use this to compute the necessary isogeny data to compute the data of the period matrices for all the Shimura curves that we construct we hope that this section may be of independent use as a working guide for applying Shimura's construction.

\section{Pirola's Hurwitz space of CyClic triple COVERS of ELLIPTIC CURVES BY GENUS FOUR CURVES}

In [Pir92] Pirola studied the space of genus four smooth curves $X$ that are Galois triple covers $p: X \rightarrow E$ of elliptic curves $E$, totally ramified over three points. We denote by $\mathcal{H}$ the Hurwitz space of such covers, which thus admits a finite map $\pi: \mathcal{H} \rightarrow \mathcal{M}_{1,3}$ and a generically injective map $\phi: \mathcal{H} \rightarrow \mathcal{M}_{4}$. Pirola's interest in $\mathcal{H}$ is in disproving a conjecture of Xiao on the fixed parts of families of curves.

Given a point $p: X \rightarrow E$ in $\mathcal{H}$ we define

$$
\operatorname{Prym}(X / E):=\operatorname{Ker}(\operatorname{Jac}(X) \rightarrow \operatorname{Jac}(E)=E)
$$

to be the (generalized) Prym variety of the covering. The polarization on $\operatorname{Prym}(X / E)$ is given by the restriction of the principal polarization from $\operatorname{Jac}(X)$, and is of type $(1,1,3)$, so that we get the Prym map $P: \mathcal{H} \rightarrow \mathcal{A}_{3,(1,1,3)}$ to the moduli space of $(1,1,3)$-polarized abelian threefolds. Using deformation theory, Pirola showed [Pir92, Section 2] that the image $P(\mathcal{H})$ is two-dimensional. Since $\operatorname{dim} \mathcal{H}=3$, the generic fiber of the map $P$ is one-dimensional. These fibers are the Kuga curves we are looking for.

Proposition 2. For any $A \in P(\mathcal{H}) \subset \mathcal{A}_{3,1,1,3)}$ the one-parameter family of four-dimensional Jacobians Jac $\left(P^{-1}(A)\right)$ is isogenous to the 
product of $A$ (as a trivial family over a base) and the universal family of elliptic curves with a suitable level structure.

Proof. For any $(p: X \rightarrow E) \in P^{-1}(A)$ the Jacobian $\operatorname{Jac}(X)$ is isogenous to the product of the fixed abelian threefold $A$ and the elliptic curve $E$, and the elliptic curve varies as we vary along the family. Thus what remains to be seen is that the family of elliptic curves that is thus obtained is indeed the universal family of elliptic curves with some level, and not some ramified cover of it.

The family of curves $y^{6}=x(x+1)(x-t)$ studied in detail in the next section is a member of this family for some $A$, in fact the period matrix will be computed below (see (4)). This family is a Shimura curve as discussed in [Möl11] and computed again below, hence for this $A$ the family of elliptic curves is as claimed.

On the other hand, the isogeny that allows to write $\operatorname{Jac}(X)$ as a product is constant over all of $P(\mathcal{H})$, since the possible isogenies are countable. This implies that the uniformization of the elliptic curve does not vary in the family over $P(\mathcal{H})$.

Corollary 3. For any $A \in P(\mathcal{H})$, the image of the one-parameter family of curves $\phi\left(P^{-1}(A)\right) \subset \mathcal{M}_{4}$ under the Torelli map Jac is a Kuga curve in $\mathcal{A}_{4}$ generically contained in the locus of Jacobians of smooth curves.

Proof. This is obvious from the preceding proposition. Alternatively, one can invoke the criterion from [MVZ12] as the universal family of abelian varieties over such a curve is globally isogenous to the product of a trivial family and the varying family of elliptic curves which reaches the Arakelov bound.

Remark 4. Note that we did not make any attempt to characterize how many times the closure of $C_{A}$ intersects the reducible locus nor how many times $C_{A}$ intersects the hyperelliptic locus. Consequently, we do not claim a priori that any of the curves $C_{A}$ reaches the Arakelov bound, just that $C_{A}$ is the intersection of a Kuga curve in $\mathcal{A}_{4}$ with $\mathcal{M}_{4}$.

Remark 5. If $A$ is a CM point, then the Kuga curve constructed above is in fact a Shimura curve - indeed, just take the elliptic curve to be $\mathrm{CM}$, and then the abelian fourfold is isogenous to a product of a CM abelian threefold and a CM elliptic curve. Thus within the twodimensional family of Kuga curves parameterized by $P(\mathcal{H})$ we have an everywhere dense collection of Shimura curves. 


\begin{tabular}{|c|l|l|l|l|l|}
\hline $\mathrm{i}$ & 1 & 2 & 3 & 4 & 5 \\
\hline $\operatorname{rank}\left(H^{1}\left(X_{t}, \mathbb{Z}\right)\right)_{\zeta^{2 i}}$ & 2 & 1 & 2 & 1 & 2 \\
\hline $\operatorname{dim}\left(H^{0}\left(X, \Omega_{X}^{1}\right)\right)_{\zeta^{2 i}}$ & 0 & 0 & 1 & 1 & 2 \\
\hline
\end{tabular}

\section{THE PERIOD MATRIX FOR THE GENUS FOUR SHIMURA-TEICHMÜLLER CURVE}

We recall that there is precisely one Shimura curve in genus 4 which happens to be also a Teichmüller curve ([Möl11]). It parametrizes the family of curves given by equations

$$
X_{t}:=\left\{y^{6}=x(x+1)(x-t)\right\},
$$

where $t$ is the parameter of the family. In this section we compute explicitly the period matrix of $X_{t}$ and then compute explicitly the isogeny between $\operatorname{Jac}\left(X_{t}\right)$ and $\operatorname{Prym}(X / E) \times E$.

Proposition 6. In the bases of homology and 1-forms defined below, the period matrix of $X_{t}$ is given by

$\left(Z_{1} \mid Z_{2}\right)=\left(\begin{array}{rrrrrrrr}\tau & \tau & 0 & -\tau-1 & 1 & 1 & 0 & -1 \\ \zeta^{2}-1 & 1 & -\zeta^{2}+1 & 1 & 1 & -\zeta^{2} & \zeta^{2} & -\zeta^{2}+1 \\ \zeta^{3}-\zeta & -\zeta^{3} & -2 \zeta^{3}+2 \zeta^{2}+\zeta-1 & \zeta^{2}-\zeta & 1 & \zeta^{2}-1 & \zeta^{3}-\zeta^{2}-2 \zeta+2 & \zeta^{2} \\ -\zeta^{3}+\zeta & \zeta^{3} & 2 \zeta^{3}+2 \zeta^{2}-\zeta-1 & \zeta^{2}+\zeta & 1 & \zeta^{2}-1 & -\zeta^{3}-\zeta^{2}+2 \zeta+2 & \zeta^{2}\end{array}\right)$

where $\zeta:=\zeta_{12}=e^{2 \pi i / 12}$ is a primitive 12-th root of unity and where $t=\lambda(\tau)$ for $\lambda: \mathbb{H} \rightarrow \mathbb{P}^{1} \backslash\{0,1, \infty\}$.

Our strategy is similar to that used by Guardia for the ShimuraTeichmüller curve in genus 3 in [Guà01, Section 3 and 4], except that our situation is somewhat more involved, as the only automorphism of a generic point of our family is $\alpha:(x, y) \mapsto\left(x, \zeta^{2} y\right)$. We thus apply the fact that most eigenspaces of the action of the automorphism on the cohomology are unitary local systems, allowing us to determine much of the period matrix from suitable special values of $t$.

Proof. We decompose the cohomology $H^{1}\left(X_{t}, \mathbb{Z}\right)$ in a direct sum of the eigenspaces for the action of $\alpha$. The eigenvalues are powers of $\zeta^{2}$, i.e. sixth roots of unity, and their dimensions can be computed by the well-known formulas for cyclic covers (see e.g. [Bou01]). 
In fact, a basis of eigenforms on $X$ is given by $\omega_{1}=d x / y^{3}, \omega_{2}=$ $d x / y^{4}, \omega_{3}=d x / y^{5}, \omega_{2}=x d x / y^{5}$. Consequently, all but the $(-1)$ eigenspace are unitary and thus the corresponding lines in the period matrix do not depend on $t$.

It suffices to compute the period matrix locally near some fixed $t_{0}$. We take $t_{0} \in \mathbb{R}_{>0}$, and let $X=X_{t_{0}}$. The first step is to define a suitable basis of $H_{1}(X, \mathbb{Z})$ and of $H^{0}\left(X, \Omega_{X}^{1}\right)$. With the branch points aligned as $\left(-1,0, t_{0}, \infty\right)$ we perform the branch cuts and draw two paths as in Figure 1. Next, w define the loops $F$ and $G$ as lifts of this paths
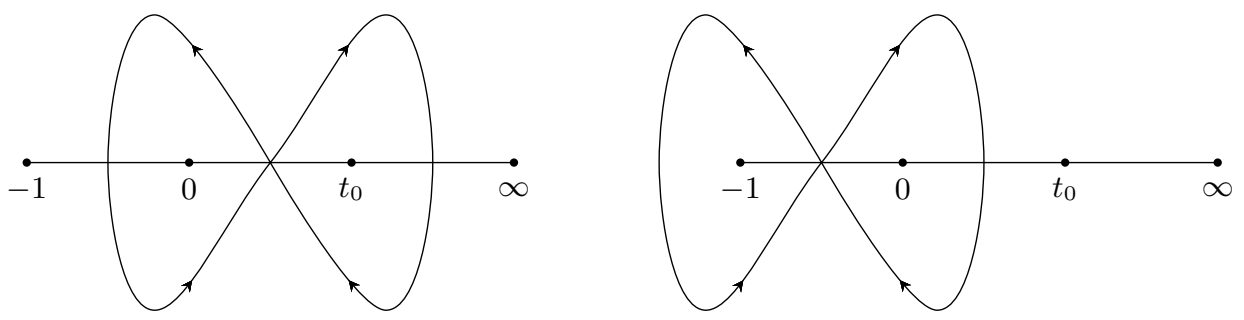

Figure 1. Paths on $\mathbb{P}^{1}(\mathbb{C})$ and ...

to $X_{t}$ as in Figure 2 with the convention that the lower left end of
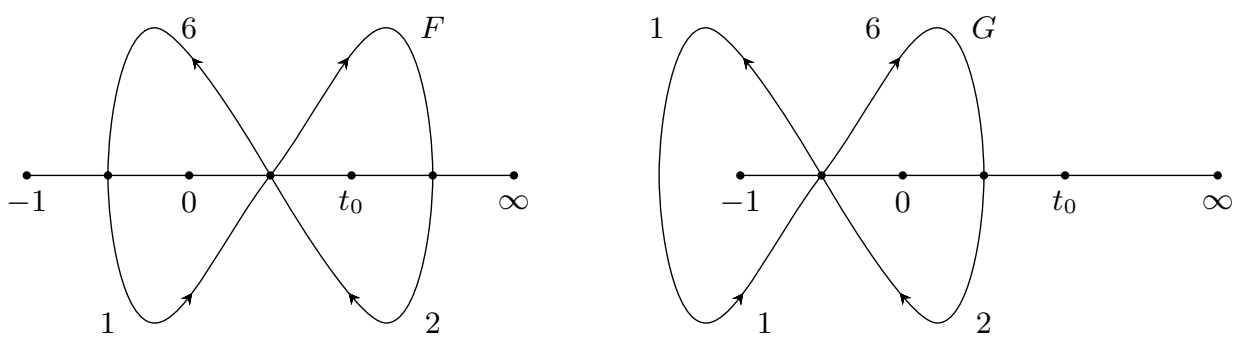

FiguRE 2. ... their lifts to $X$.

the 'butterfly' is always on sheet number one. 1 The set of paths $\left\{u_{1+k}=\alpha^{k}(F), u_{7+\ell}=\alpha^{\ell}(G), k, \ell=0, \ldots, 5\right\}$ has intersection matrix

\footnotetext{
${ }^{1}$ We follow the sheet numbering convention that clockwise loops around $-1,0$ and $t$ are given by the permutation $\gamma=(123456)$, while the loop around $\infty$ is given by $\gamma^{-3}=(14)(25)(36)$. The action of $\alpha$ corresponds to a clockwise turn and thus decreases the sheet number by one. We also use the convention that the intersection number is plus one if the intersection is pointing as the fingers of the right hand.
} 
$M=\left\langle u_{i}, u_{j}\right\rangle$ given by

$$
M=\left(\begin{array}{rrrrrrrrrrrr}
0 & -1 & 0 & 0 & 0 & 1 & -1 & 1 & 0 & 0 & 0 & 0 \\
1 & 0 & -1 & 0 & 0 & 0 & 0 & -1 & 1 & 0 & 0 & 0 \\
0 & 1 & 0 & -1 & 0 & 0 & 0 & 0 & -1 & 1 & 0 & 0 \\
0 & 0 & 1 & 0 & -1 & 0 & 0 & 0 & 0 & -1 & 1 & 0 \\
0 & 0 & 0 & 1 & 0 & -1 & 0 & 0 & 0 & 0 & -1 & 1 \\
-1 & 0 & 0 & 0 & 1 & 0 & 1 & 0 & 0 & 0 & 0 & -1 \\
1 & 0 & 0 & 0 & 0 & -1 & 0 & -1 & 0 & 0 & 0 & 1 \\
-1 & 1 & 0 & 0 & 0 & 0 & 1 & 0 & -1 & 0 & 0 & 0 \\
0 & -1 & 1 & 0 & 0 & 0 & 0 & 1 & 0 & -1 & 0 & 0 \\
0 & 0 & -1 & 1 & 0 & 0 & 0 & 0 & 1 & 0 & -1 & 0 \\
0 & 0 & 0 & -1 & 1 & 0 & 0 & 0 & 0 & 1 & 0 & -1 \\
0 & 0 & 0 & 0 & -1 & 1 & -1 & 0 & 0 & 0 & 1 & 0
\end{array}\right) .
$$

Since the $8 \times 8$ minor of the intersection matrix corresponding to $u_{1}, u_{2}, u_{3}, u_{4}, u_{7}, u_{8}, u_{9}, u_{10}$ is non-degenerate, it follows that these paths generate $H_{1}(X, \mathbb{Z})$. We compute that the homology classes

$e_{1}:=u_{1}, \quad e_{2}:=u_{3}, \quad e_{3}:=u_{1}-u_{3}+u_{5}+u_{6}, \quad e_{4}:=u_{2}-u_{5}-u_{8}$

$e_{5}:=u_{7}, \quad e_{6}:=u_{9}, \quad e_{7}:=u_{2}+u_{3}-u_{5}+u_{7}, \quad e_{8}:=u_{1}+u_{2}+u_{4}+u_{6}$

form a symplectic basis for $H_{1}(X, \mathbb{Z})$.

To compute the period matrix in this basis, it suffices to determine $f_{i}=\int_{F} \omega_{i}$ and $g_{i}=\int_{G} \omega_{i}$. since $\alpha$ acts on $\omega_{i}$ by a power of $\zeta$ and since

$$
\int_{\alpha^{k}(F)} \omega_{i}=\int_{F} \alpha^{-k}\left(\omega_{i}\right)
$$

First, we use the intermediate 3 -to- 1 covering of $\mathbb{P}^{1}$ by the family of elliptic curves $\mathcal{E}_{t}: y^{3}=x(x+1)(x-t)$. This covering is a constant family of elliptic curves, since there is no ramification at infinity. We let $q: \mathcal{X}_{t} \rightarrow \mathcal{E}_{t},(x, y) \mapsto\left(x, y^{2}\right)$ be the quotient map. The holomorphic one-form on $\mathcal{E}_{t}$ is $\omega_{E}=d x / y^{2}$ and its pullback is $q^{*} \omega_{E}=\omega_{2}$. On $E$ we may suppose $t=1$ and then there is an additional automorphism $\beta_{E}:(x, y) \mapsto(-x, \zeta y)$ besides $\alpha_{E}:(x, y) \mapsto\left(x, \zeta^{2} y\right)$. We view $\mathcal{E}_{t}$ as a three-sheeted covering of the projective line and decompose each sheet into two half-planes, which we number and then label $U$ and $L$ for upper and lower. Then $\beta_{E}$ maps $1 U$ to $1 L$, maps $1 L$ to $3 U$, and so on - consistently with the fact that $\beta_{E}^{2}=\alpha_{E}$ decreases the sheet number by one. Now denoting $F_{E}:=q(F)$ and $G_{E}:=q(G)$, we have $\beta_{E}\left(G_{E}\right)=-\alpha_{E}^{-1}\left(F_{E}\right)$ and $\beta_{E}\left(F_{E}\right)=-\alpha_{E}^{2}\left(G_{E}\right)$. Hence

$$
\int_{F_{E}} \omega_{E}=-\int_{\beta\left(G_{E}\right)} \alpha_{E}^{*} \omega_{E}=-\zeta^{-4} \int_{G_{E}}\left(\beta^{-1}\right)^{*} \omega_{E}=\zeta^{-2} \int_{G_{E}} \omega_{E}
$$

and consequently $g_{2}=\int_{G} q^{*} \omega_{E}=\int_{G_{E}} \omega_{E}=\zeta^{2} \int_{F} \omega_{2}=\zeta^{2} f_{2}$ gives us the row in the period matrix corresponding to integrals of $\omega_{2}$

The holomorphic one-form $\omega_{1}$ is the pullback of the one-form from the non-isotrivial family of elliptic curves $y^{2}=x(x+1)(x-t)$. We keep $f_{1}$ and $g_{1}$ as indeterminates and determine the rest of the first row using (1). 
Finally, $\omega_{3}$ and $\omega_{4}$ belong to a unitary local system and we may calculate their periods at any special point. We choose $t=1$, where $\mathcal{X}_{1}$ has the extra automorphism $\beta:(x, y) \mapsto(-x, \zeta y)$ with $\beta^{2}=\alpha$. Similarly to the situation for $\mathcal{E}_{t}$, we see that $\beta$ maps the sheets by the pattern $1 U \mapsto 1 L \mapsto 6 U \mapsto 6 L \mapsto \cdots$. Consequently, $\beta(G)=-\alpha^{-1}(F)$ (and $\beta(F)=-\alpha^{2}(G)$ ). We thus get

$$
f_{3}=\int_{F} \omega_{3}=-\int_{\beta(G)} \alpha^{*} \omega_{3}=-\zeta^{-5} \int_{G}\left(\beta^{-1}\right)^{*} \omega_{3}=\zeta^{-5} \int_{G} \omega_{3}
$$

and $f_{4}=-\zeta^{-5} g_{4}$ due to an extra minus sign appearing when pulling back by $\beta^{-1}$. Another application of the same argument implies that for our special point $t=1$ we have $f_{4}=\zeta^{-3} g_{4}$.

The previous calculations also imply that we may normalize the differentials so that $f_{i}=1$ for $i=1,2,3,4$. We let $\tau=g_{1}$ and altogether the period matrix with respect to the $\omega$ and $\left\{e_{1}, \ldots, e_{8}\right\}$ is as stated in the proposition.

While the automorphism $\alpha$ of order 6 may not deform to any genus four curves outside the family $X_{t}$ above (and from our description it would in fact follow that it does not, but we will not need this result), the automorphism $\varphi=\alpha^{2}$ of order 3 by definition does deform to the Hurwitz space $\mathcal{H}$. Thus in what follows the cover $p: X \rightarrow E$ given by $(x, y) \mapsto\left(x, y^{3}\right)$ that is obtained as the quotient by $\varphi$ will be most important to us.

We want to exhibit an isogeny $\operatorname{Jac}(X) \rightarrow \operatorname{Prym}(X / E) \times E$. On the level of one-forms, this is easy, since the first row of the period matrix $\left(Z_{1} \mid Z_{2}\right)$ corresponds to $\omega_{1}$, which is $\varphi$-invariant. Moreover, we identify the period lattice $\Lambda$ of $\operatorname{Jac}(X)=\mathbb{C}^{4} / \Lambda$ with $\mathbb{Z}^{8}$ and consider the sublattices $\Lambda_{E}$ resp. $\Lambda_{P}$ of $\Lambda$ that are orthogonal to the real and imaginary part of the second through fourth row (resp. first row) of $\left(Z_{1} \mid Z_{2}\right)$. Considering $\Lambda_{E}$ as a sublattice of $\mathbb{Z}^{8}$ and writing its components as the first and fifth column of a base change matrix $B$, and proceeding similarly with $\Lambda_{P}$, we obtain the matrix

$$
B:=\left(\begin{array}{rrrrrrrr}
1 & 0 & -1 & -1 & 1 & 0 & 1 & -2 \\
1 & 0 & 1 & 2 & 1 & 0 & 0 & 1 \\
0 & 1 & 0 & 0 & 0 & 0 & 0 & 0 \\
-1 & 0 & 0 & 1 & -1 & 0 & 1 & -1 \\
0 & 0 & 0 & 1 & 1 & 0 & 0 & 0 \\
0 & 0 & 0 & 1 & 1 & 0 & 1 & 0 \\
0 & 0 & 0 & 0 & 0 & 1 & 0 & 0 \\
1 & 0 & 0 & 1 & 0 & 0 & 0 & 1
\end{array}\right)
$$

such that

$$
\left(Z_{1} \mid Z_{2}\right) B=\left(\begin{array}{cccc}
3 \tau & 0 & 3 \tau+3 & 0 \\
0 & Z_{1}^{(3)} & 0 & Z_{2}^{(3)}
\end{array}\right)
$$


is indeed the period matrix of the product of $E$ and the period matrix of $\operatorname{Prym}(X / E)$, which has period matrix $\left(Z_{1}^{(3)} \mid Z_{2}^{(3)}\right)$ given by

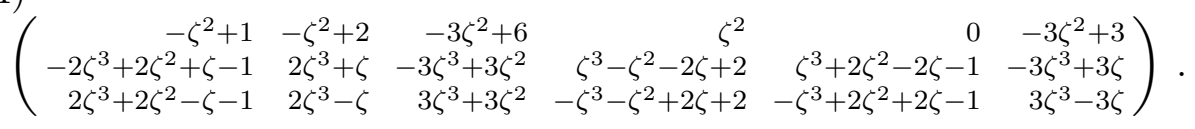

We check that the polarization $J_{3}=B^{T} J B$, where $J$ is the standard principal polarization matrix with ones on the diagonal of the upper right block, is indeed of type $(1,1,3)$. Furthermore, we see that the $(1,1,3)$ polarized abelian threefold with the period matrix $\left(Z_{1}^{(3)} \mid Z_{2}^{(3)}\right)$ indeed admits indeed an order three automorphism, preserving the polarization $J_{3}$, given by the diagonal matrix $\operatorname{diag}\left(\zeta^{4}, \zeta^{8}, \zeta^{8}\right)$ acting on the left and the inverse of

$$
M_{3}:=\left(\begin{array}{rrrrrr}
0 & 0 & 0 & -1 & 0 & 0 \\
0 & 1 & 0 & 0 & -3 & 3 \\
0 & 0 & 1 & 0 & 1 & 0 \\
1 & 0 & 0 & -1 & 0 & 0 \\
0 & 0 & -3 & 0 & -2 & 0 \\
0 & -1 & -3 & 0 & 0 & -2
\end{array}\right)
$$

acting on the right.

\section{FAMilies of ABELIAN THREefolds With COMPLEX MULTIPLICATION}

The moduli spaces of abelian varieties with given endomorphism ring and polarization, nowadays called PEL-Shimura varieties, have been constructed by Shimura in [Shi63]. This construction is presented in the textbook [BL04, Chapter 9], the notation of which we follow, and to which we refer for further details.

In the previous section we dealt with the Shimura-Teichmüller curve $\mathcal{X}$ the general Jacobian in which has a $\mathbb{Z} / 6$ automorphism. Our goal now is to explicitly construct $(1,1,3)$-polarized abelian threefolds with an order three automorphism inducing complex multiplication by $\mathbb{Z}[\rho]$ with $\rho:=\eta^{4}$ a third root of unity. The moduli space of polarized abelian threefolds with such an endomorphism is two-dimensional.

Proposition 7. There exists an irreducible component of the moduli space of $(1,1,3)$ polarized abelian threefolds with a $\mathbb{Z} / 3$ automorphism such that the $3 \times 6$ period matrices are $\left(Z_{1}^{(3)}\left(z_{1}, z_{2}\right) \mid Z_{2}^{(3)}\left(z_{1}, z_{2}\right)\right.$ for a suitable choice of basis, where the matrix $Z_{1}^{(3)}$ is equal to

$$
\left(\begin{array}{rrr}
3^{-1 / 4}\left(\zeta^{2}+3 \zeta+1\right) z_{2} & \left(\zeta^{2}+3 \zeta+1\right)\left(z_{1}+1\right) & \left(-\zeta^{3}+8 \zeta+3\right)\left(z_{1}+1\right) \\
-2 \zeta^{3}+2 \zeta^{2}+\zeta-1 & 3^{3 / 4}\left(\zeta^{3}+\zeta^{2}-1\right) z_{2}-3 \zeta^{2}\left(z_{1}-1\right) & a_{23} \\
2 \zeta^{3}+2 \zeta^{2}-\zeta-1 & 3^{3 / 4}\left(\zeta^{3}+\zeta^{2}-1\right) z_{2}+\left(4 \zeta^{3}+2 \zeta^{2}-5 \zeta-4\right)\left(z_{1}-1\right) & a_{33}
\end{array}\right)
$$

where

$$
a_{23}=3^{3 / 4}\left(3 \zeta^{3}+3 \zeta^{2}-2 \zeta-1\right) z_{2}-3\left(\zeta^{2}-3 \zeta-1\right)\left(z_{1}-1\right)
$$


and

$$
a_{33}=3^{3 / 4}\left(3 \zeta^{3}+3 \zeta^{2}-\zeta-4\right) z_{2}+\left(10 \zeta^{3}+\zeta^{2}-17 \zeta-11\right) z_{1}+\left(8 \zeta^{3}+2 \zeta^{2}-13 \zeta-11\right) ;
$$

while the matrix $Z_{2}^{(3)}$ is equal to

$$
\left(\begin{array}{rrr}
3^{-1 / 4}\left(3 \zeta^{3}+\zeta^{2}-3 \zeta-2\right) z_{2} & \left(3 \zeta^{3}+\zeta^{2}-3 \zeta-2\right)\left(z_{1}+1\right) & b_{13} \\
\zeta^{3}-\zeta^{2}-2 \zeta+2 & 3\left(z_{1}+1\right)+3^{3 / 4}\left(\zeta^{3}-\zeta+1\right) z_{2} & b_{23} \\
-\zeta^{3}-\zeta^{2}-2 \zeta+2 & 3^{3 / 4}\left(-\zeta^{3}+\zeta+1\right) z_{2}+\left(\zeta^{3}+2 \zeta^{2}+4 \zeta+2\right)\left(z_{1}+1\right) & b_{33}
\end{array}\right)
$$

where

$$
\begin{aligned}
& b_{13}=\left(8 \zeta^{3}+3 \zeta^{2}-7 \zeta-3\right) z_{1}+10 \zeta^{3}+6 \zeta^{2}-8 \zeta-6 \\
& b_{23}=\left(9 \zeta^{3}-3\right) z_{1}-3^{3 / 4}\left(12 \zeta^{3}-3 \zeta^{2}-9 \zeta+9\right) z_{2}+9 \zeta^{3}-3 \zeta^{2} \\
& b_{33}=\left(\zeta^{3}+10 \zeta^{2}+10 \zeta+7\right) z_{1}-3^{3 / 4}\left(\zeta^{3}-\zeta^{2}-3 \zeta-3\right) z_{2}+5 \zeta^{3}+5 \zeta^{2}+8 \zeta+2
\end{aligned}
$$

for $\left(z_{1}, z_{2}\right)$ in some complex 2 -ball. Moreover, the $3 \times 6$ period matrix obtained for

$$
z_{1}=\frac{-2 \zeta^{3}+\zeta^{2}+\zeta-3}{2}, \quad z_{2}=3^{-1 / 4} \frac{\zeta^{3}-2 \zeta^{2}+1}{2} .
$$

is precisely equal to the matrix (4) obtained in the previous section.

Once we have proven this proposition, our main result follows immediately

Proof of Theorem 1. Indeed, once we have determined the period matrices $\left(Z_{1}^{(3)}\left(z_{1}, z_{2}\right) \mid Z_{2}^{(3)}\left(z_{1}, z_{2}\right)\right)$ of the Pryms appearing in our construction, to obtain the period matrices of the genus four Jacobians that form Shimura curves, we will substitute them into the form of period matrices given by (3) and undo the base change by the matrix $B$ given by $(2)$.

Thus it remains to prove the proposition using Shimura's construction. However, the construction of universal families by Shimura involves quite a number of choices. So this section is a guide to the practical use of Shimura's construction: how to adapt the choices so that a given period matrix appears as a member of the family.

Proof of Proposition 7. For the proof, we follow Shimura's original construction, adapted and specialized to our case, as explained in [BL04, Chapter 9], to which we refer for all justifications of the steps. In our case the endomorphism ring is the maximal order $\mathbb{Z}[\rho]$ in the field $K=\mathbb{Q}(\rho)$, while the Rosati involution is simply the complex conjugation, and its fixed is $K_{0}=\mathbb{Q}$, of which $K$ is thus a quadratic extension. Abelian threefolds with this endomorphism ring are given as quotients of the 2-ball

$$
B_{2}:=\left\{z=\left(\begin{array}{c}
z_{1} \\
z_{2}
\end{array}\right) \in \mathbb{C}^{2}:\left|z_{1}\right|^{2}+\left|z_{2}\right|^{2}<1\right\}
$$


Following Shimura, to construct all such abelian threefolds (for complex multiplication by any order in $K)$ one chooses any pair $(\mathcal{M}, T)$ consisting of a free rank 6 submodule $\mathcal{M}$ of $K^{3}$ and a non-degenerate signature $(2,1)$ matrix $T \in \operatorname{Mat}_{3 \times 3}(K)$ such that $\bar{T}^{T}=-T$, satisfying

$$
\operatorname{tr}_{\mathbb{Q}}^{K}\left(a^{T} T \bar{b}\right) \in \mathbb{Z} \quad \text { for all } a, b \in \mathcal{M} .
$$

To construct such $(\mathcal{M}, T)$, recall that the signature $(2,1)$ condition means that there exists a matrix $W \in \mathrm{GL}_{3}(\mathbb{C})$ satisfying

$$
T=W^{T}\left(\begin{array}{cc}
I_{2} & 0 \\
0 & -i
\end{array}\right) \bar{W},
$$

where we denote by $I_{2}$ the product of $i$ and the $2 \times 2$ identity matrix. To any $z \in B_{2}$ we associate the linear map $J_{z}: \mathbb{C}^{6} \rightarrow \mathbb{C}^{3}$ given by left multiplication by the matrix

$$
J_{z}:=\left(\begin{array}{cc}
\left(z^{t}, 1\right) W & 0 \\
0 & \left(I_{2}, z\right) \bar{W}
\end{array}\right)
$$

and we let $j: \mathcal{M} \rightarrow \mathbb{C}^{6}$ be given by one embedding of $K \rightarrow \mathbb{C}$ in the first three coordinates and the complex conjugate embedding in the remaining three coordinates. Then the abelian variety $X_{z}:=\mathbb{C}^{3} / J_{z}(j(\mathcal{M}))$ with the polarization

$$
H:=\left(\begin{array}{cc}
|z|^{-1} & 0 \\
0 & \left(I_{2}-\bar{z} z^{T}\right)^{-1}
\end{array}\right)
$$

has the desired endomorphism, since by a direct matrix computation one checks that

$$
a J_{z}(j(b))=J_{z}(j(a b)) \text { for all } a \in K \quad \text { and } b \in \mathcal{M},
$$

and hence the action of $K$ on $\mathbb{C}^{3}$ is compatible with the lattice $\Lambda=$ $J_{z}(j(\mathcal{M}))$ (see [BL04, Chapter 9.3]). Moreover, $\operatorname{Im} H$ is indeed an integer-valued bilinear form on $\Lambda$, as one checks by computing

$$
(\operatorname{Im} H)\left(J_{z}(j(a)), J_{z}(j(b))\right)=\operatorname{tr}_{\mathbb{Q}}^{K}\left(a^{T} T \bar{b}\right)
$$

for all $a, b \in \mathcal{M}$.

Consequently, our goal is to find a pair $(\mathcal{M}, T)$ such that the period matrix $\left(Z_{1}^{(3)} \mid Z_{2}^{(3)}\right)$ of the Shimura-Teichmüller curve, given by (4), appears in the family of period matrices $J_{z}(j(\mathcal{M}))$ for some $\left(z_{1}, z_{2}\right) \in B_{2}$. To find $\mathcal{M}$, note that since $\left(Z_{1}^{(3)} \mid Z_{2}^{(3)}\right)$ has complex multiplication by a maximal order, we must have $\mathcal{M} \cong \mathbb{Z}[\rho]^{3}$. Hence we have to pick three elements in $\mathbb{Z}^{6}$ that together with their $\rho$-images (with $\rho$ acting by left multiplication by the matrix $M_{3}$ given in (5), computed for the special case) generate $\mathbb{Z}^{6}$. A choice of such elements is given for example by $u_{1}=e_{1}, u_{2}=e_{2}+e_{5}, u_{3}=2 e_{3}+e_{5}-e_{6}$, and $u_{3+k}=\rho u_{k}$ for $k=1,2,3$. 
Let now $L$ be the base change matrix from $\mathbb{Z}^{6}$ to the basis $u_{1}, \ldots, u_{6}$. We compute

$$
L^{T} J_{3} L=\left(\begin{array}{rrr}
0 & 0 & 0 \\
0 & 0 & 1 \\
0 & -1 & 0
\end{array}\right), \quad L^{T} M_{3}^{T} J_{3} L=\left(\begin{array}{rrr}
-1 & 0 & 0 \\
0 & 0 & 1 \\
0 & 2 & 9
\end{array}\right)
$$

Now to make a choice of $T$, we use (7) and the fact that $\operatorname{Im} H$ is the intersection form on $\Lambda$. The matrix

$$
T=\left(\begin{array}{rrr}
\frac{2}{3} \zeta^{4}+\frac{1}{3} & 0 & 0 \\
0 & 0 & -\zeta^{4} \\
0 & -\zeta^{4}-1 & -6 \zeta^{4}-3
\end{array}\right)
$$

has the desired property $(7)$, since $\operatorname{tr}_{\mathbb{Q}}^{K}(T+\bar{T})=L^{T} J_{3} L$ and since $\operatorname{tr}_{\mathbb{Q}}^{K}\left(\zeta^{4} T+\overline{\zeta^{4} T}\right)=L^{T} M_{3}^{T} J_{3} L$. We can now find a $W$ satisfying (6), and we choose

$$
W=\left(\begin{array}{rrr}
0 & 3-\zeta & 0 \\
3^{-1 / 4} & 0 & 0 \\
0 & 1 & 3-i
\end{array}\right) \text {. }
$$

Substituting all of these choices, for Shimura's form of the period matrices we finally obtain

$$
\begin{aligned}
& \left(Z_{1}^{(S)}\left(z_{1}, z_{2}\right) \mid Z_{2}^{(S)}\left(z_{1}, z_{2}\right)\right)= \\
& \left(\begin{array}{rrrrrr}
3^{-1 / 4} z_{2} & z_{1}+1 & (3-\zeta) z_{1}+3-i & 3^{-1 / 4} \zeta^{4} z_{2} & \zeta^{4}\left(z_{1}+1\right) & \left((3-\zeta) z_{1}+3-i\right) \zeta^{4} \\
0 & z_{1}+1 & (3-i)\left(z_{1}+1\right)+3-\zeta^{-1} & 0 & \left(z_{1}+1\right) \zeta^{8} & \left((3-i)\left(z_{1}+1\right)+\left(3-\zeta^{-1}\right)\right) \zeta^{8} \\
3^{-1 / 4} & z_{2} & (3+i) z_{2} & 3^{-1 / 4} \zeta^{8} & \zeta^{8} z_{2} & (3+i) \zeta^{8} z_{2}
\end{array}\right)
\end{aligned}
$$

Finally, the last indeterminacy in the choice of the period matrices is that we can choose different eigenforms within the two eigenspaces. This means we can further have a base change matrix $C=\left(c_{i j}\right)$ for holomorphic one-forms and are looking for $z=\left(z_{1}, z_{2}\right)$ such that

$$
\left(\begin{array}{ccc}
c_{11} & 0 & 0 \\
0 & c_{22} & c_{23} \\
0 & c_{32} & c_{23}
\end{array}\right)\left(Z_{1}^{(S)}(z) \mid Z_{2}^{(S)}(z)\right)=\left(Z_{1}^{(3)} \mid Z_{2}^{(3)}\right) .
$$

This system has a unique solution, given by

$$
\begin{array}{lll}
c_{11}=\zeta^{2}+3 \zeta+1, & c_{22}=-3 \zeta, & c_{23}=3^{3 / 4}\left(\zeta^{3}-\zeta^{2}+1\right), \\
c_{32}=4 \zeta^{3}+2 \zeta^{2}-5 \zeta-4, & c_{33}=3^{3 / 4}\left(\zeta^{3}+\zeta^{2}-1\right)
\end{array}
$$

and $\left(z_{1}, z_{2}\right)$ as stated in the proposition. With these values of $c_{i j}$, the left hand side of (8) is the family given in the statement of the proposition.

\section{REFERENCES}

[BL04] C. Birkenhake and H. Lange. Complex Abelian Varieties, volume 302 of Grundlehren der Mathematischen Wissenschaften. Springer-Verlag, Berlin, second edition, 2004.

[Bou01] I. Bouw. The p-rank of ramified covers of curves. Compositio Math., 126(3):295-322, 2001.

[CLZ15] K. Chen, X. Lu, and K Zuo. A note on Shimura subvarieties in the hyperelliptic Torelli locus , 2015, arXiv:math.AG/1504.05380. 
[FGP14] P. Frediani, A. Ghigi, and M. Penegini. Shimura varieties in the Torelli locus via Galois coverings, 2014, arXiv: math.AG/1402.0973, Intern. Math. Res. Notices, to appear.

[FPP15] P. Frediani, M. Penegini, and P. Porru. Shimura varieties in the Torelli locus via Galois coverings of elliptic curves, 2015, arXiv: math.AG/1508.00730, Geom. Dedicata, to appear.

[GM13] S. Grushevsky and M. Moeller. Shimura curves within the locus of hyperelliptic Jacobians in genus three, 2013, arXiv:math.AG/1308.5155, to appear in Intern. Math. Res. Notices.

[Guà01] J. Guàrdia. Explicit geometry on a family of curves of genus 3. J. London Math. Soc. (2), 64(2):299-310, 2001.

[LZ13] X. Lu and K Zuo. On Shimura curves in the Torelli locus of curves, 2013, arXiv:math.AG/1311.5858.

[MO13] B. Moonen and F. Oort. The Torelli locus and special subvarieties. In Handbook of moduli. Vol. II, volume 25 of Adv. Lect. Math. (ALM), pages 549-594. Int. Press, Somerville, MA, 2013.

[Möl11] M. Möller. Shimura and Teichmüller curves. J. Mod. Dyn., 5(1):1-32, 2011.

[MVZ12] M. Möller, E. Viehweg, and K. Zuo. Stability of Hodge bundles and a numerical characterization of Shimura varieties. J. Differential Geom., 92(1):71-151, 2012.

[Oor97] F. Oort. Canonical liftings and dense sets of CM-points. In Arithmetic geometry (Cortona, 1994), Sympos. Math., XXXVII, pages 228-234. Cambridge Univ. Press, Cambridge, 1997.

[Pir92] G. P. Pirola. On a conjecture of Xiao. J. Reine Angew. Math., 431:75-89, 1992.

[Shi63] G. Shimura. On analytic families of polarized abelian varieties and automorphic functions. Ann. of Math. (2), 78:149-192, 1963.

Mathematics Department, Stony Brook University, Stony Brook, NY 11794-3651, USA.

E-mail address: sam@math.sunysb.edu

Institut für Mathematik, Goethe-Universität Frankfurt, RobertMaYer-Str. 6-8 60325 Frankfurt am Main, Germany

E-mail address: moeller@math.uni-frankfurt.de 